
ANISAKIDI IN SPECIE ITTICHE MARINE PESCATE NEL NORD SARDEGNA

\title{
MOLECULAR CHARACTERIZATION OF ANISAKID NEMATODES IN FISHES OF NORTHERN SARDINLANSEA
}

Tedde T. ${ }^{1}$, Piras M.C. ${ }^{2}$, Merella P. ${ }^{2}$, Mele P. ${ }^{1}$, Lorenzoni G. ${ }^{1}$, Rosa M.N. ${ }^{1}$, Salza S. ${ }^{1}$, Assaretti A. ${ }^{1}$, Santoru F. ${ }^{1}$, Marongiu E. ${ }^{1}$, Virgilio S. ${ }^{1}$

${ }^{1}$ Istituto Zooprofilattico Sperimentale della Sardegna, Dipartimento di Igiene degli Alimenti, Sassari; ${ }^{2}$ Università di Sassari, Dipartimento di Biologia Animale, Sezione di Parassitologia e Malattie Parassitarie

SUMMARY

KEYWORDS
The authors report results of analysis carried out during 2008-2010 for identification and molecular characterization of larval Anisakis nematodes isolated from fishes of the northern Sardinian sea.

parasitic zoonosis, Anisakis pegreffii, Anisakis physeteris, PCR-RFLP.

L'anisakidosi è una zoonosi parassitaria causata da larve di nematodi appartenenti a diversi generi della famiglia Anisakidae, fra cui Anisakis, Contracaecum e Pseudoterranova. Gli stadi larvali di Anisakis sp. rappresentano un potenziale rischio per la salute umana (1) in seguito al consumo di ospiti paratenici, quali pesci marini o molluschi cefalopodi crudi, poco cotti, salati, leggermente marinati o affumicati a freddo. Tra i mesi di ottobre 2008 e giugno 2010 sono stati esaminati complessivamente 869 campioni appartenenti a 8 specie di teleostei, di cui 138 Merluccius merluccius, 79 Micromesistius poutassou, 62 Phycis blennoides, 374 Sardina pilchardus, 40 Sardinella aurita, 45 Scomber japonicus, 77 Trachurus mediterraneus, 54 Trachurus trachurus. I campioni esaminati sono stati sottoposti ad esame visivo ed osservazione allo stereomicroscopio, seguito da digestione enzimatica del tessuto muscolare. $\mathrm{Nel}$ corso dell'indagine sono state complessivamente isolate 2619 larve di Anisakidi, di cui 942 appartenenti al genere Hysterothylacium e 1677 al genere Anisakis. Le larve del genere Anisakis isolate sono state differenziate in tipo I (1625) e tipo II (52) mediante osservazione al microscopio ottico ed una parte di queste $(50 \%)$ è stata sottoposta ad analisi biomolecolare per l'identificazione della specie mediante l'amplificazione di una porzione della regione genomica nucleare ITS con l'utilizzo dei primers forward APEF per A. pegreffii e APHF per A. physeteris e del primer reverse NC2 per entrambe le specie (2). In una seconda fase è stata effettuata una ulteriore identificazione di specie delle larve del genere Anisakis mediante PCRRFLP (3). L'utilizzo di primers specie-specifici ha consentito di identificare come $A$. pegreffii tutte le larve del Tipo I esaminate (812), con un prodotto di amplificazione di $577 \mathrm{bp}$ e come $A$. physeteris tutte le larve del Tipo II, con un amplificato di $637 \mathrm{bp}$. La metodica PCR-RFLP ha confermato i risultati ottenuti, in quanto in base alla combinazione dei patterns RFLP tutte le forme larvali del Tipo I sono risultate appartenenti alla specie $A$. pegreffii e tutte le larve del Tipo II alla specie $A$. physeteris.

\section{BIBLIOGRAFIA}

1. Pampiglione S., Rivasi F., Criscuolo M., De Benedettis A., Gentile A., Russo S., Testini M., Villani M. (2002). Human Anisakiasis in Italy: A report of Eleven New Cases. Pathology Research and Practice 198: 429-434.

2. Fang W., Xu S., Zhang S., Wang Y., Chen X., Luo D. (2010) Multiple primer PCR for the identification of anisakid nematodes 
from Taiwan Strait. Experimental Parasitology 124: 197-201.

3. D'Amelio S., Mathiopulos KD., Santos CP., Pugachev ON., Webb SC., Picanco MO., Paggi L. (2000). Genetic markers in ri- bosomial DNA for the identification of members of the genus Anisakis defined by PCR-based restriction fragment length polymorphism. International Journal Parasitology 30: 223-226 